\title{
Science Metrics Systems in Biomedical Sciences: Current Trends
}

\section{Enver Zerem ${ }^{1,2}$}

\section{ABSTRACT}

The publication of scientific research is principally information published in scientific journals and they are cornerstone of knowledge dissemination, as well as an essential criterion for academic and scientific evaluation, recruiting funds and career progression. However, it is very difficult to apply right measures and scientific criteria which can objectively assess scientific research. For many years there has been a great interest in scientific ranking and evaluation of scientific journals, but also of scientific contribution of scientists. It is generally accepted that the IF (WoS) and the total number of citations of articles published in the journal, are the most relevant parameters of the journal's significance. However, the scientific significance of a scientist is much more complicated to evaluate and the value of their scientific production cannot be directly reflected by the importance of the journals in which their articles are published.

The majority of existing science metric systems, which evaluate the achievement of scientists are focused solely on the number of citations of their articles. Based on a long term of academic experience, the author describes and evaluates the most known scientific databases which are used in biomedical sciences. Also, the author proposes Z-score as a new science metric system which takes into account the current IF (WoS) and total number of citations of the journal in which the article is published, as well as author's contribution to the scientific article. In that way, proposed criteria greatly remedy major discrepancies in evaluating scientific production of individual authors and institutions.

Key words: science metrics systems, scientific impact factor, Z-score, number of citations, author contribution.

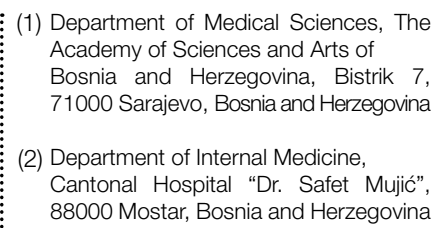

Correspondence:

ENVER ZEREM

E: zerem@live.com

T: +38761138789

ARTICLE INFO

Recived: $26^{\text {th }}$ December 2018 Accepted: $17^{\text {th }}$ January 2019
Society invests significant resources in biomedical research, seeking for ways to improve human health. However, the efficiency of society's investments in biomedical research, in terms of improved health outcomes, has not been fully confirmed. Social and scientific importance and the quality of scientific research highly depend on the usefulness of the research results. The scarcity of scientific research funds and the tendency to direct the funds towards high-quality research accentuates the importance of measuring and assessing the quality of research and knowledge valorization. However, it is very difficult to apply the right measures and scientific criteria which can objectively assess scientific research ${ }^{1-3}$.
The product of scientific research is principally information published in scientific journals and they are cornerstone of knowledge dissemination, as well as an essential criterion for academic and scientific evaluation, recruiting funds and career progression 4,5 . It is true that, beside evaluation of scientific publications, there is a wide range of other scientific activities which also reflect scientific credibility such as ${ }^{5}$ :

- number and quality of extramural grants,

- leadership in national or international academic societies,

- service on editorial boards of respected journals,

- service on government sponsored national peer review committees, 
- the number of $\mathrm{PhD}$ students delivered,

- the amount of coverage of one's scientific output in the lay press, etc.

Although, those activities are important and give certain significance to the scientific credibility of a scientist, the relevant science metrics systems only cover publications, and omit other criteria of scientific relevance, which are typically used in judging promotions and tenure of scientists. The reason for this is the fact that these activities, regardless of their importance, are very heterogeneous since each of them has specific characteristics and requires different parameters for evaluation. Hence, for these parameters of scientific relevance there are no universal evaluation criteria and their value is mainly assessed individually depending on the purpose of the assessment ${ }^{5,6}$.

\section{The ranking of scientists and scientific journals}

For many years there has been a great interest in scientific ranking and evaluation of scientific journals, but also of scientific contribution of scientists. The most known scientific databases which are used in biomedical sciences are:

Current Contents (CC)

- Web of Science (WoS)

- Journal Citation Reports (JCR)

- Index Medicus, Medline, PubMed

- Excerpta Medica (EMBASE)

- Scopus,

- Schoolar,

- H-index

Most of those scientific bases (except for Scopus, Scholar, H-index and WoS which rank scientists) present and rank scientific journals only.

\subsection{Current Contents (CC)}

Current Contents is a platform for Clarivate Analytics (formerly Thomson Reuters). It is the most appreciated database that is usually available through the Web of Science. The reasons for its popularity are the relatively high journal selection criteria, its coverage of all areas of science, the update frequency, the author's summary, the author's address, the names and addresses of the publisher, and its ability to review the content of a particular issue of the journal. Current Contents is published in seven following sections (each containing more than 1000 sources):

- Current Contents / Agriculture, Biology \& Environmental Sciences
- Current Contents / Arts \& Humanities

- Current Contents / Clinical Medicine

- Current Contents / Engineering, Computing \& Technology

- Current Contents / Life Sciences

- Current Contents / Physical, Chemical \& Earth Sciences

- Current Contents / Social \& Behavioral Sciences

Through above noted seven sections Current Contents covers all areas of science. Most biomedical journals are included in the Current Contents/Clinical Medicine section, but some biomedical journals are classified under the sections of Current Contents/Life Sciences and Current Contents/Social \& Behavioral Sciences ${ }^{5,6}$.

\subsection{Web of Science (WoS)}

The Web of Science is a platform for Clarivate Analytics, which provides access to quoted databases covering all areas of science. The platform contains more than 33,00o indexed journals and nearly one billion records of quoted references, and includes articles, conference proceedings, reports, patents, and more. The following databases are available through the Web of Science interface ${ }^{6,7}$ :

- Science Citation Index Expanded

- Social Sciences Citation Index

- Arts \& Humanities Citation Index

- Emerging Sources Citation Index

- Book Citation Index - Science

- Book Citation Index - Social Sciences \& Humanities

- Conference Proceedings Citation Index- Science

- Current Chemical Reactions

- ESCI Backfiles

- Index Chemicus

\subsection{Journal Citation Reports (JCR)}

On the basis of the data obtained from the citation databases (Science Citation Index and Social Science Citation Index) Eugene Garfield created a special statistical database and named it Journal Citation Reports (JCR). At the end of June each year, Clarivate Analytics publishes the JCR list for the previous year. Impact factor (IF) is a quantitative aid for ranking, evaluating, categorizing and comparison of the journals. IF is calculated as an quotient when dividing the number of citations received during the year that refer to articles from the previous two years. It is gen- 
erally accepted that IF and the total number of citations of all articles published in the journal represent a relevant measure of their value and scientific influence. For some domains, it is much more relevant to have an IF for a 5 -year period rather than for a standard 2-year period ${ }^{8,9}$.

\subsection{Index Medicus, Medline, PubMed}

Index Medicus is the print version of the bibliographic citation database of the National Library of Medicine, and MEDLINE is its online counterpart. PubMed is an online database that provides access to citations in MEDLINE as well as those from additional life sciences journals. MEDLINE and PubMed are among the most popular and widely used literature databases for health care professionals. Nearly 4500 journals are indexed in MEDLINE, and even more are indexed in PubMed ${ }^{10}$.

\subsection{Excerpta Medica (EMBASE)}

Embase is a highly versatile, multipurpose and up-to-date biomedical research database. Produced by Elsevier, it covers the most important international biomedical literature containing over 32 million records from over 8,500 currently published journals from 1947 to the present. Embase's international coverage expands across biomedical journals from 95 countries and is available through a number of database vendors ${ }^{9}$.

\subsection{Scopus}

Scopus is a quotation database that indexes sources from all over the world, and includes more than 69 million records for 21,950 reviewed journals, 280 trade journals from almost all fields of science, more than 560 series of books, 8 million conference proceedings and more than 39 million patents (Wikipedia).

\subsection{H-index}

Almost all relevant scintimetric indexes which evaluate the achievement of scientists are focused on the number of citations of their articles. The best-known scintimetric system which assesses the individual scientific contribution of scientists is the so-called $\mathrm{H}$-index which is calculated as the lowest ranked article which number of citations matches its ranking number (for example, a scientist whose $\mathrm{H}$-index is 10 must have at least 10 articles which have 10 or more citations).

However, H-index has considerable shortcomings because the system is based solely on the evaluation of the number of individual article ci- tations. Therefore, H-index obviously favors older articles which are available for quotation for a longer time, and negatively impacts on assessment of scientific value of new articles and scientific production of perspective scientists. Besides, $\mathrm{H}$-index does not take into account the individual contribution of each author in an evaluated article, since according to $\mathrm{H}$-index all authors of an article are treated as equal. Hence, $\mathrm{H}$-index does not tackle the ever-present problem of expanding author lists with authors whose contribution may be minute or none ${ }^{11,12}$.

\subsection{Other scientific database}

There are several other databases such as: Google Schoolar, PageRank index; Altmetrics; g-index; e-index; i-index; total publications; total number of citations etc $9,13,14$.

\section{Does Zerem-score bring improvements?}

The existence of such a large number of science metric systems shows that there is no perfect scientific metric index that accurately measures the scientific contribution of scientists and scientific journals. It is generally accepted that the IF (WoS) and the total number of citations of articles published in the journal, are the most relevant parameters of the journal's significance. The scientific significance of a scientist is much more complicated to evaluate than that of a scientific journal, since the scientific production value cannot be directly reflected by the importance of the journal in which the article is published ${ }^{5}$.

As a director for scientific research in the institution where I used to work every year I was in charge of annual assessment of the employees' scientific contribution. At the beginning, it seemed like a simple job. The scientific contribution was ranked on the basis of IF of the journal in which the article was published. However, some authors had published more articles throughout the year. There were different types of articles which were published in journals indexed in different scientific bases. Also, those articles were new, therefore they had not been cited by other authors. It is known that, the number of citations of a particular article, as the relevant measure of the value of that article, has its limitations since it requires excessive time lag and gives advantage to older articles of similar quality. Furthermore, the contribution of all the authors in a scientific article is usually not the same. Therefore, it is very difficult to apply the right measures and scientific criteria which 
can objectively assess a new scientific research and provide precise qualitative and quantitative data on which new articles could be evaluated. Based on the long term experience, I was proposing the new criteria (named Z-score) which can objectively estimate the scientific effect of scientists and institutions. The Z-score criteria have been published in the Journal of Biomedical Informatics $5,12,15$.

According to the Z-score criteria the overall scientific score of an author is calculated as the sum of two scores (author contribution score-ACoS and author citation contribution score-ACCS). $\mathrm{ACoS}$ is calculated as the scientific value of the journal in which an article is published and the authors' specific contribution in this article. ACCS is calculated as the scientific values of the particular article expressed through the number of quotations that this article has received and the authors' specific contribution in the article. In order to successfully apply and calculate $\mathrm{Z}$-score, we have created the adequate computer software - Z-score calculator, which encompasses all the parameters described in the proposed criteria. Also, Z-score calculator is designed to be compatible with all browsers and it is capable of automatic collection of data once linked to a browser ${ }^{5,15}$.

\section{CONCLUSIONS}

Whether scientists like it or not, the societal impact of their research is an increasingly important factor in their academic and scientific evaluation, recruiting public funds for scientific research and career progression [5]. This has always been the case, but current trends in scientific and academic community increasingly emphasize the need to improve criteria and establish measures that can objectively assess the societal impact of research and would provide better qualitative and quantitative data which will enable the societal and scientific community to objectively assess the value of scientists and scientific research. This is especially important in developing countries where a complex interrelation between politics and the academic community significantly impacts on the process of acquisition of scientific and academic titles 5, 16, 17.

However, it is very difficult to apply right measures and scientific criteria which can objectively assess scientific research, providing precise qualitative and quantitative data on which funding agencies could base their decisions. The existence of a large number of science metric systems shows that there is no perfect scientific metric index that can accurately measure the scientific contribution of scientists and scientific journals. It is true that the current science metric systems have multiple shortcomings and are not ideal for an objective assessment of scientific research and the scientists' significance. However, without the introduction and application of internationally recognized scientific criteria in the evaluation of scientific research, and the coordination of academic progress in accordance with these criteria, there is room left for the decision makers within the academic community to lower the criteria margin to the level which they subjectively consider relevant, without complying with the internationally recognized criteria. Therefore, the application of internationally recognized scientific criteria in the evaluation of scientific research is necessary and these criteria should be constantly improved. I consider Z-score criteria sustainable and capable of objective estimation of the scientific effect of scientists and institutions. I hope this article contributes to the discussion about science metric systems, raising questions and motivating the expression of different viewpoints with the intention to improve science metric systems and make them more objective and competent in the complex process of evaluating scientific production in biomedical research. 


\section{REFERENCES}

1. Dixon AK. Publishing and academic promotion. Singapore Med J 2009; 50: 847-50.

2. Bowen A, Casadevall A. Increasing disparities between resource inputs and outcomes, as measured by certain health deliverables, in biomedical research. Proc Natl Acad Sci USA 2015; 112: 11335-40.

3. Bornmann L, Haunschild R, Marx W. Policy documents as sources for measuring societal impact: how often is climate change research mentioned in policy-related documents? Scientometrics. 2016; 109: 1477-95.

4. Van Noorden R. Metrics: a profusion of measures. Nature 2010; 465: 864-6.

5. Zerem E. The ranking of scientists based on scientific publications assessment. J Biomed Inform 75; 2017 : 107-9.

6. Thwaites T. Research metrics: calling science to account. Nature 2014; 511: S57-S6o.

7. Stonehill HI. Science Citation Index: information retrieval by propinquity. Hem Ind 1965; 10: 416-7.

8. Garfeld E. Citation indexes to science: a new dimension in documentation through association of ideas. Science 1955; 122: 108-11.

9. Abbott A, Cyranoski D, Jones N, et al. Metrics: do metrics matter? Nature 2010; 465: 860-2.

10. Tisdale JE. Indexing of Journals in MEDLINE, PubMed, and Other Bibliographic Databases-Why Not CJHP? Can J Hosp Pharm 2004; 57: 77-8.

11. Hirsch JE. An index to quantify an individual's scientific research output. Proc Natl AcadSci USA 2005; 102: 16569-72.

12. Zerem E. The ranking of scientists. J Biomed Inform 2018; 79:147-8.

13. Sugimoto CR, Larivière V. Altmetrics: broadening impact or amplifying voices? ACS Cent Sci 2017; 3: 674-6.

14. Gao C, Wang Z, Li X, Zhang Z, Zeng W. PR-index: using the h-index and PageRank for determining true impact. PLoS One 2016; 11 (9): eo161755,

15. Zerem E, Kunosić S. The ranking of scientists: Computational calculation of Z-score. J Biomed Inform 2018; 81: 133-4.

16. Zerem E. Right criteria for academia in Bosnia and Herzegovina. Lancet 2013; 382: 128.

17. Zerem E. Academic community in a developing country: Bosnian realities. Med Glas (Zenica) 2014; 11: 248-51. 